\title{
First in Human Experience of the Performance of the New 5.5-LP Size Zephyr Endobronchial Valve
}

\author{
Karin Klooster Marlies van Dijk T. David Koster Dirk-Jan Slebos \\ Department of Pulmonary Diseases, UMCG Groningen Research Institute for Asthma and COPD, \\ University of Groningen, Groningen, The Netherlands
}

\section{Keywords}

Emphysema $\cdot$ Endobronchial valve $\cdot$ Lung volume reduction

\begin{abstract}
Background: Bronchoscopic lung volume reduction using the Zephyr ${ }^{\circledR}$ endobronchial valve (EBV) is a guideline treatment for patients with advanced emphysema. To achieve volume reduction, it is crucial that there is absence of collateral ventilation and a complete occlusion of the target lobe. While 3 EBV sizes (4.0; 4.0-LP; and 5.5) are currently available to accommodate all airway sizes, local anatomical variations sometimes warrant a valve with a wide diameter but shorter length. To address this, a new "low profile" 5.5-LP EBV has been introduced. Objective: In this study, we evaluated the feasibility, safety, and efficacy of this new 5.5-LP EBV. Methods: This was a single-center, prospective, open-label study. Patients were included if eligible for valve treatment with a local anatomy suitable to place at least one 5.5-LP EBV. Feasibility of placement of the 5.5-LP EBV was reported. Safety, CT parameters, pulmonary function tests, and St. George's Respiratory Questionnaire (SGRQ) were assessed at baseline and 6 weeks after treatment. Results: We included 30 patients with severe chronic obstructive pulmonary disease (forced expiratory volume in $1 \mathrm{~s}$ [FEV $] 29 \pm 10 \%$; [RV] $242 \pm$ $46 \%$; and SGRQ $56 \pm 11$ points). Besides the regular EBV sizes, a median of 1 (1-3) of the new 5.5-LP EBV was placed. No
\end{abstract}

valve adjustment was needed during the initial procedure. A single asymptomatic small pneumothorax was observed in 1 patient. In 4 patients, a revision bronchoscopy was performed due to absence of clinical benefit. In 1 patient, this was related to a dislocation of the 5.5-LP EBV. Clinically relevant improvements were seen in target lobar volume reduction $(-1,554 \mathrm{~mL}), \mathrm{FEV}_{1}+39 \%, \mathrm{RV}-960 \mathrm{~mL}$, and SGRQ -18 points. Conclusions: In this first in human study, the 5.5-LP EBV could be placed into wide segments with a shorter landing length without unexpected complications and with good efficacy outcomes.

(C) 2019 The Author(s)

Published by S. Karger AG, Basel

\section{Introduction}

Bronchoscopic lung volume reduction using one-way endobronchial valves (EBV; Zephyr; Pulmonx Corporation, CA, USA) is a guideline treatment for patients with advanced emphysema [1] and can lead to improvement in airflow obstruction, hyperinflation, exercise tolerance, physical activity [2], and quality of life [3-7]. To achieve the desired volume reduction, it is crucial that there is proven absence of collateral ventilation and a complete occlusion with valves of the treatment target lobe [8].

The EBV is a nitinol self-expandable retainer with a silicone coating and valve mechanism which is broncho- 
Fig. 1. Available Zephyr $^{\circledR}$ endobronchial

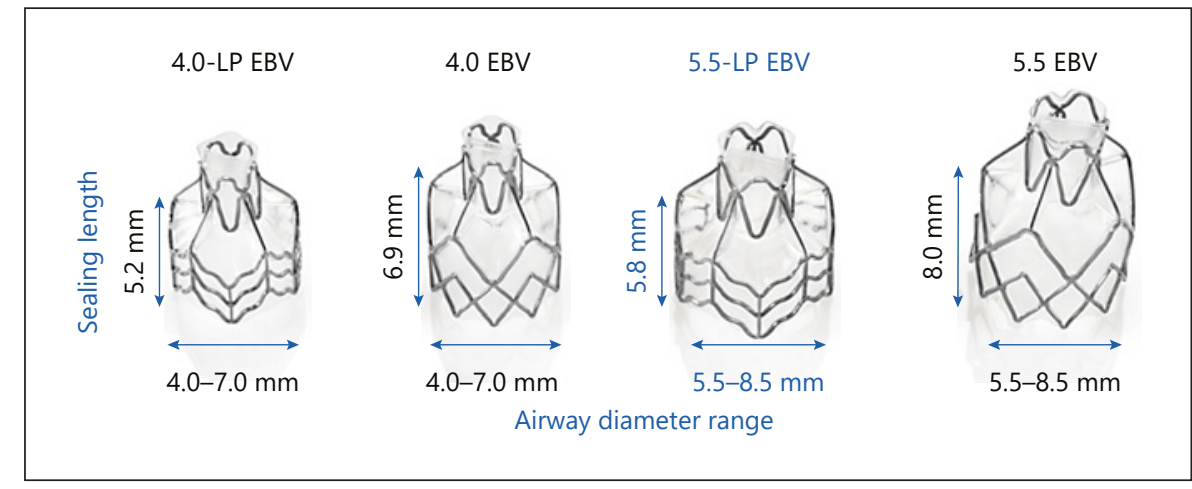
valves

scopically delivered to the (sub)segments of the target lobe [9]. Multiple valves are required to fully occlude the entire target lobe. Until recently, the EBV was available in 3 different sizes. The available sizes 4.0 EBV and 4.0-LP EBV are to be placed in bronchial lumens ranging from 4.0 to $7.0 \mathrm{~mm}$ in diameter, with the sealing length of the valve of the 4.0-LP EBV being 25\% shorter in length than the 4.0 EBV to accommodate shorter airway lengths. The $5.5 \mathrm{EBV}$ is to be placed in bronchial lumens ranging from 5.5 to $8.5 \mathrm{~mm}$ in diameter and with a necessary sealing length of $8.0 \mathrm{~mm}$. These 3 valve designs accommodate most airway anatomical variations. However, especially in the apical segments (both B1 and B6), a 5.5 EBV diameter range but with a shorter sealing length than $8.0 \mathrm{~mm}$ is sometimes needed and might facilitate easier procedures (see Fig. 1 for available Zephyr ${ }^{\circledR}$ EBV).

To address this need, a new "low profile" 5.5-LP EBV (sealing length $5.8 \mathrm{~mm}$ instead of the "regular" $8 \mathrm{~mm}$ length; diameter range $5.5-8.5 \mathrm{~mm}$ ) has been introduced to accommodate shorter airway lengths in the targeted bronchial segment. While the sizes 4.0 EBV, 4.0-LP EBV, and 5.5 EBV have been available on the European market for many years, the 5.5-LP EBV has been introduced in September 2018 (see Figure 1 for available Zephyr ${ }^{\circledR}$ EBV).

In this study, we tested the procedural performance of the new 5.5-LP EBV for the first time.

\section{Methods}

This was a single-center, prospective, open-label study. We included patients with chronic obstructive pulmonary disease (COPD) and severe emphysema who were scheduled for EBV treatment and who received at least one new "low profile" 5.5-LP EBV. All patients in this study provided written consent, were treated in our hospital, and were registered in a national treatment registry (BREATH-NL: NCT02815683).

\section{EBV Treatment}

EBV treatment was performed using a flexible therapeutic bronchoscope under general anesthesia as described before [9]. Prior to valve placement, the Chartis assessment was performed to determine collateral ventilation in the target lobe. Patients with absence of collateral ventilation and with a local anatomy suitable to place at least one 5.5-LP EBV to achieve complete occlusion of the target lobe were included in this study. Lobar occlusion was performed in a single sequence, and the valves were (sub)segmentally placed.

\section{Follow-Up}

The feasibility of initial placement of the 5.5-LP EBV was reported. Safety was monitored. High-resolution CT, postbronchodilator pulmonary function tests, and SGRQ were assessed at baseline and 6 weeks after EBV treatment. Quantitative CT analysis was performed using Thirona's Lung Quantification (Thirona, Nijmegen, The Netherlands) on the baseline CT scan and the 6-week follow-up scan. Repeat bronchoscopy was performed if the patient did not experience clinical benefit at the 45-day follow-up visit and if the CT scan suggested a possible valve dislocation.

\section{Statistical Analysis}

Data are shown as median values (minimum to maximum) or as means \pm SD. The Wilcoxon signed rank test was used to compare results before and 6 weeks after EBV treatment. IBM SPSS statistics 23 (IBM, New York, NY, USA) was employed for statistical analysis.

\section{Results}

From September 2018 to May 2019, thirty COPD patients with severe emphysema treated with regular EBVs and at least one 5.5-LP EBV were included in this analysis. See Table 1 for demographics and baseline characteristics.

\section{EBV Treatment}

A median of 4 valves (range 2-6) were placed per patient, with a median of 1 (range 1-3) of the new 5.5-LP EBVs. In these 30 patients, a total of 41 new 5.5-LP EBVs 
were placed without additional valve adjustments or periprocedural replacements during the procedure. Procedural information is provided in Table 2.

\section{Efficacy}

Six weeks (50 \pm 13 days) after EBV treatment, there was significant $(p<0.001)$ clinically relevant improvement with respect to baseline levels regarding target lobar volume reduction, residual volume (RV), forced expiratory volume in $1 \mathrm{~s}\left(\mathrm{FEV}_{1}\right)$, and SGRQ score. The median relative change in target lobar volume reduction on $\mathrm{CT}$ scan was $-100 \%$ (range from -100 to $-27 \%$ ), in $\mathrm{FEV}_{1}$ $+285 \mathrm{~mL}$ (range from +70 to $+870 \mathrm{~mL}$ ), in $\mathrm{RV}-22 \%$ (range from -46 to $+16 \%$ ), and absolute median change in the SGRQ total score was -18 points (range from -38 to +12 points). See Figure 2 for effectiveness outcomes and responder rates.

\section{Revision Bronchoscopy}

At the 6-week follow-up visit, 4 patients did not have clinical benefit, and therefore a revision bronchoscopy was performed. In 1 patient, the lack of effect was related to dislocation of a 5.5-LP EBV. This patient had a clinically relevant lung volume reduction ( $\mathrm{RV}$ was reduced by $870 \mathrm{~mL}) 3$ months after revision bronchoscopy. See Table 3 for reasons for revision bronchoscopy.

\section{Safety}

No adverse events occurred during the initial EBV treatment and during revision bronchoscopy. In 30 patients, 1 (3\%) small nonsymptomatic apical pneumothorax was observed 4 days after treatment which did not require chest tube drainage. No other adverse events occurred in this study.

\section{Discussion}

This is the first study investigating the procedural performance of the new 5.5-LP Zephyr EBV to bronchoscopically achieve lobar occlusion in patients with advanced emphysema and proven absence of collateral ventilation measured with Chartis.

We found that placement of this new size valve is feasible. During the 30 initial valve placement procedures, using 41 new EBVs, no valve adjustments, replacements, or repositioning was required. In this study, the 5.5-LP valve was predominantly placed in the apical segments (LB6) due to a shorter length of the (sub)segment; however, it appeared that all segments allow an easier treat-

Table 1. Demographics and baseline characteristics $(n=30)$

Age, years

Body mass index, $\mathrm{kg} / \mathrm{m}^{2}$

$\mathrm{FEV}_{1}, \%$ of predicted

FVC, \% of predicted

$\mathrm{FEV}_{1} / \mathrm{FVC}, \%$

$\mathrm{RV}, \%$ of predicted

RV/TLC, \%

$\mathrm{DL}_{\mathrm{CO}}, \%$ of predicted

SGRQ total score, points

Target lobe volume, $\mathrm{mL}$

Target lobe voxel density <-950 HU, \%
Gender, females/males

TLC, $\%$ of predicted

$61 \pm 8$

$21 / 9$

$24 \pm 4$

$29 \pm 10$

$71 \pm 14$

$32 \pm 8$

$140 \pm 14$

$242 \pm 46$

$63 \pm 6$

$32 \pm 10$

$56 \pm 11$

$2,042 \pm 679$

$52 \pm 11$

Data are shown as means $\pm \mathrm{SD}$. $\mathrm{FEV}_{1}$, forced expiratory volume in $1 \mathrm{~s}$; FVC, forced vital capacity; TLC, total lung capacity; RV, residual volume; DLCO, carbon monoxide diffusing capacity; SGRQ, Saint George Respiratory Questionnaire.

Table 2. Endobronchial valve (EBV) procedures and results

\begin{tabular}{lc}
\hline EBV treatment & $(n=30)$ \\
Mean duration (range), min & $11(5-25)$ \\
Target lobe for EBV treatment, $n(\%)$ & \\
Right upper lobe & $2(7)$ \\
Right upper + middle lobe & $4(13)$ \\
Right lower lobe & $2(7)$ \\
Left upper lobe & $4(13)$ \\
Left lower lobe & $18(60)$ \\
Median EBV placed/patient, $n$ (range) & $4(2-6)$ \\
EBV size 4.0 & $1(0-3)$ \\
EBV size 4.0-LP & $0(0-2)$ \\
EBV size 5.5 & $1(0-3)$ \\
EBV size 5.5-LP & $1(1-3)$ \\
Segments treated with 5.5-LP EBV, $n$ & $(n=41)$ \\
RB1 & 3 \\
RB2 & 2 \\
RB3 & 1 \\
RB4/5 & 1 \\
RB6 & 3 \\
RB9/10 & 2 \\
LB1/2 & 1 \\
LB3 & 4 \\
LB6 & 14 \\
LB8 & 4 \\
LB9 & 3 \\
LB8/9/10 & 2 \\
\hline
\end{tabular}

ment using this new valve design (Table 2). Placing a "regular 5.5 EBV" at these positions would have been suboptimal due to a too short "landing zone (length)"; alternatively, the 5.5-LP EBV-treated positions could have been treated using 2 , 3 , or even 4 smaller valves instead. 


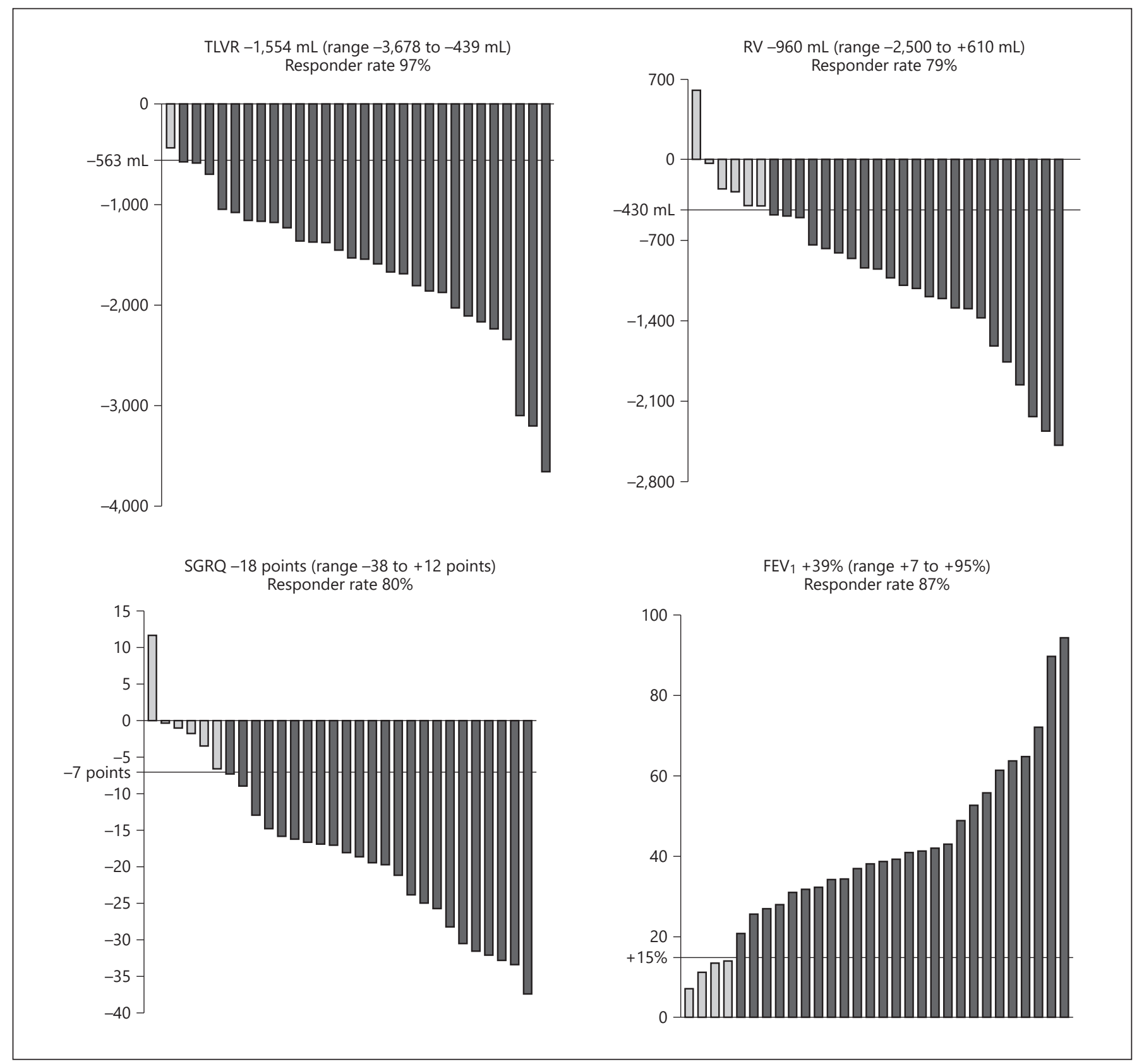

Fig. 2. Effectiveness outcomes and responder rates. Effectiveness outcomes in absolute change (TLVR, RV, and SGRQ) and in relative change $\left(\mathrm{FEV}_{1}\right)$ from baseline to follow-up 6 weeks after EBV placement. Data are shown as median values (minimum to maximum). All $p<0.001$. Wilcoxon signed rank test was used to calculate differences. Minimal clinically important difference (MCID)

We were able to successfully place the new 5.5-LP EBV in all segments where needed and together with the additional valves placed to achieve a successful lobar occlusion of the target lobe.

First in Human Experience with the New 5.5-LP Zephyr EBV for TLVR was $\geq 563 \mathrm{~mL}$ reduction [10]; relevant change for $\mathrm{FEV}_{1}$ $\geq 15 \%$ improvement; $\mathrm{MCID}$ for $\mathrm{RV} \geq 430 \mathrm{~mL}$ reduction [11]; and MCID for SGRQ $\geq 7$ points reduction [12]. It was not possible to obtain reliable body plethysmography measurement in 1 patient, with a TLVR on CT scan of $-1,372 \mathrm{~mL} 6$ weeks after EBV treatment.

Total lobar occlusion of the treatment target lobe was achieved in all patients resulting in a large $(>1,554 \mathrm{~mL})$ target lobe volume reduction exceeding far the minimal clinically important difference [10]. Despite this signifi- 
Table 3. Reasons for revision bronchoscopy $(n=4)$

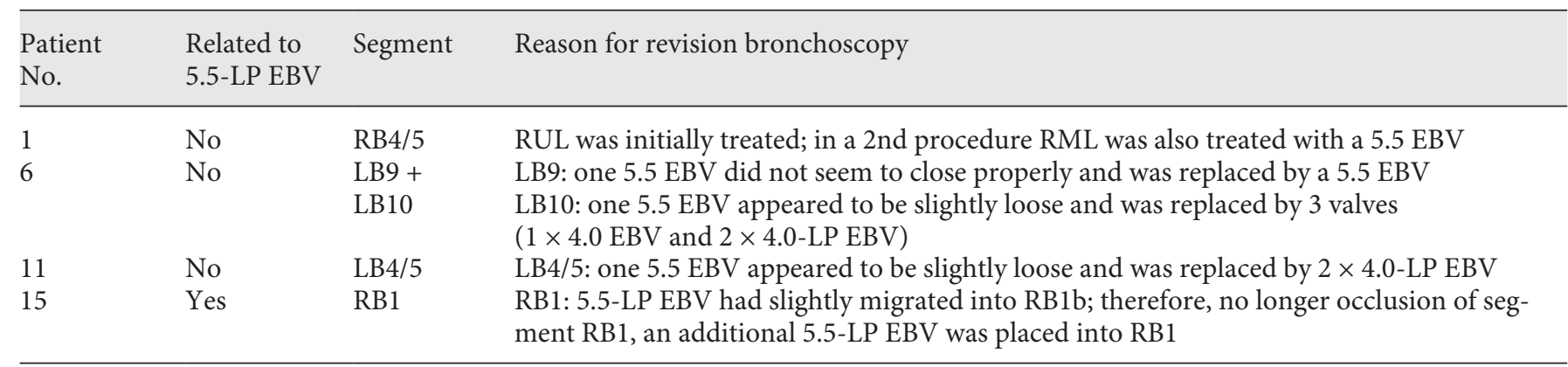

RUL, right upper lobe; RML, right middle lobe; EBV, Zephyr endobronchial valve.

cant lung volume reduction, remarkably we only observed a single nonsymptomatic small pneumothorax (rate of $3.3 \%$ in $n=30$ patients treated). This rate is considerably lower than the rates reported in recent randomized controlled trials of the Zephyr EBV [3-5]. It is rather speculative why this rate is so low, while the outcome is optimal in this patient group. First, the majority (67\%) was treated in the lower lobes, where in general a lower rate of pneumothoraxes occur compared to upper lobes. Second, we extubated the patients while they are still deeply sedated with strong cough suppression for the first hour after treatment (intravenous lidocaine and opiates). Our patients are actively mobilized the morning after the procedure and discharged after at least 3 days of clinical observation.

A limitation of this study is the short follow-up period of 6 weeks after treatment, which may not be sufficient to evaluate any late valve migrations. Our experience from previous studies [3-5] shows that approximately $10 \%$ valve migrations occur in the first 12 months after initial EBV treatment. Therefore, a longer follow-up is needed to evaluate the occurrence of migration of the 5.5-LP EBV.

\section{Conclusion}

In this first in human study, the Zephyr 5.5-LP EBV is shown to be a viable option for placement in segments of wider diameter but shorter landing space. Total occlusion of the EBV treatment target lobe was successful in all patients. No unexpected adverse events occurred. Six weeks after EBV treatment, there was clinically relevant improvement in target lobar volume reduction on CT scan and in lung function outcomes. Long-term follow-up is needed to evaluate sustained effects.

\section{Statement of Ethics}

All patients in this study provided written consent, were treated in our hospital; and were registered in a national treatment registry (BREATH-NL: NCT02815683). According to the ethics committee of our hospital; this study did not fall within the scope of the WMO (Dutch Medical Research with Human Subjects Law) and therefore formal approval was not needed.

\section{Disclosure Statement}

K.K. and D.-J.S. received travel grants and financial support from PulmonX as consultant not relating to this trial. T.D.K. and M.D. have no conflicts of interest. PulmonX was not involved in drafting of the manuscript but had the right to review the manuscript in its final form regarding publication of proprietary information. No information was deleted.

\section{Author Contributions}

All authors had complete access to the data, and reviewed and approved the manuscript.
References

Respiration 2020;99:50-55 DOI: $10.1159 / 000504182$
1 Global Initiative for Chronic Obstructive G Global strategy for the diagnosis, management and prevention of chronic obstructive pulmonary disease 2018 report. 2018.

2 Hartman JE, Klooster K, Slebos DJ, Ten Hacken NH. Improvement of physical activity after endobronchial valve treatment in emphysema patients. Respir Med. 2016 Aug;117: $116-21$.

3 Klooster K, ten Hacken NH, Hartman JE, Kerstjens HA, van Rikxoort EM, Slebos DJ. Endobronchial Valves for Emphysema without Interlobar Collateral Ventilation. N Engl J Med. 2015 Dec;373(24):2325-35. 
4 Kemp SV, Slebos DJ, Kirk A, Kornaszewska M, Carron K, Ek L, et al. A Multicenter Randomized Controlled Trial of Zephyr Endobronchial Valve Treatment in Heterogeneous Emphysema (TRANSFORM). Am J Respir Crit Care Med. 2017 Dec;196(12):1535-43.

5 Criner GJ, Sue R, Wright S, Dransfield M, Rivas-Perez $\mathrm{H}$, Wiese $\mathrm{T}$, et al. A Multicenter Randomized Controlled Trial of Zephyr Endobronchial Valve Treatment in Heterogeneous Emphysema (LIBERATE). Am J Respir Crit Care Med. 2018 Nov; 198(9):1151-64.

6 Herth FJ, Eberhardt R, Gompelmann D, Ficker JH, Wagner M, Ek L, et al. Radiological and clinical outcomes of using Chartis ${ }^{\mathrm{TM}}$ to plan endobronchial valve treatment. Eur Respir J. 2013 Feb;41(2):302-8.
7 Valipour A, Slebos DJ, Herth F, Darwiche K, Wagner M, Ficker JH, et al.; IMPACT Study Team. Endobronchial valve therapy in patients with homogeneous emphysema. Results from the IMPACT study. Am J Respir Crit Care Med. 2016 Nov;194(9):1073-82.

8 van Geffen WH, Slebos DJ, Herth FJ, Kemp SV, Weder W, Shah PL. Surgical and endoscopic interventions that reduce lung volume for emphysema: a systemic review and meta-analysis. Lancet Respir Med. 2019 Apr;7(4):313-24.

9 Slebos DJ, Shah PL, Herth FJ, Valipour A. Endobronchial Valves for Endoscopic Lung Volume Reduction: Best Practice Recommendations from Expert Panel on Endoscopic Lung Volume Reduction. Respiration. 2017;93(2): $138-50$.
10 Welling JB, Hartman JE, van Rikxoort EM, Ten Hacken NH, Kerstjens HA, Klooster K, et al. Minimal important difference of target lobar volume reduction after endobronchial valve treatment for emphysema. Respirology. 2018 Mar;23(3):306-10.

11 Hartman JE, Ten Hacken NHT, Klooster K, Boezen HM, De Greef MHG, Slebos D-J. The minimal important difference for residual volume in patients with severe emphysema. EurRespirJ.2012;40(5).DOI:10.1183/09031936. 00219111

12 Welling JBA, Hartman JE, Ten Hacken NHT, Klooster K, Slebos D-J. The minimal important difference for the St George's Respiratory Questionnaire in patients with severe COPD. Eur Respir J. 2015 DOI: 10.1183/13993003. 00535-2015. 